BMJ Open

Ophthalmology

\title{
Economic modelling of immediately sequential bilateral cataract surgery (ISBCS) in the National Health Service based on possible improvements in surgical efficiency
}

\author{
David P O'Brart (D) ,1,2 Harry Roberts, ${ }^{1,2}$ Khayam Naderi, ${ }^{1,2}$ Jack Gormley ${ }^{1}$
}

\begin{abstract}
To cite: 0 'Brart DP, Roberts $\mathrm{H}$, Naderi K, et al. Economic modelling of immediately sequential bilateral cataract surgery (ISBCS) in the National Health Service based on possible improvements in surgical efficiency. BMJ Open Ophthalmology 2020;5:e000426. doi:10.1136/ bmjophth-2019-000426
\end{abstract}

Received 7 December 2019 Revised 14 May 2020 Accepted 17 May 2020
Check for updates

(C) Author(s) (or their employer(s)) 2020. Re-use permitted under CC BY-NC. No commercial re-use. See rights and permissions. Published by BMJ.

${ }^{1}$ Guy's and Saint Thomas' NHS Foundation Trust, London, UK ${ }^{2}$ King's College London, London, UK

Correspondence to Dr David P 0'Brart; davidobrart@aol.com

\section{ABSTRACT}

Objective To test a hypothesis that operating room (OR) productivity in the National Health Service (NHS) can be improved with the introduction of immediately sequential bilateral cataract surgery (ISBCS).

Methods and analysis Previously published time and motion data of 140 unilateral cataract surgeries conducted at five different NHS locations were reanalysed to construct a hypothetical model where only ISBCS ( \pm one unilateral case) were conducted while maintaining time durations of all key tasks previously studied. Possible time efficiency savings were calculated for the ISBCS model and percentage increases in numbers of eyes operated per 4-hour theatre session calculated. Gains in efficiency were correlated with factors from the baseline data to predict which settings could improve efficiency most by undertaking ISBCS.

Results Based on remodelling our time and motion study (TMS) data as hypothetical ISBCS cases, we could expect a mean $16 \%$ reduction (range $9.8 \%-17.8 \%$ ) in the time taken for two cataract operations, translating into a mean $54 \%$ improvement (range 38\%-67\%) in number of cases currently performed per list and an $18 \%$ improvement (range 9\%-28\%) even if the number of unilateral cases per list had been fully maximised. An average number of four ISBCS cases per list (range 3-6) were required to achieve sufficient time savings to allow an extra unilateral surgery to be conducted.

Conclusion The introduction of routine ISBCS has the potential to improve the productivity of cataract surgery within the NHS, with efficiencies being possible in both high-volume and low-volume surgical models.

\section{INTRODUCTION}

Small incision phacoemulsification cataract surgery is one of the most successful medical interventions currently undertaken, with minimal complications and generally excellent refractive and visual outcomes. ${ }^{1}$ It has been reported that individuals with bilateral cataracts, who undergo surgery in both rather than in only one of their eyes, have better improvements in visual function and quality of life. ${ }^{2}$ Traditionally, and typically, only one eye

\section{Key messages}

What is already known about this subject?

- Immediately sequential bilateral cataract surgery (ISBCS) is safe when conducted with appropriate aseptic precautions and the use of intracameral antibiotics.

ISBCS is cost-effective compared with unilateral surgery in terms of hospital expenditure, patient travel, paid home care costs and loss of working times.

- Time and motion study can be used to highlight factors to improve surgical efficiency within the health sector.

\section{What are the new findings?}

ISBCS has the potential to improve the productivity of cataract surgery within the National Health Service.

Efficiencies are possible in both high-volume and low-volume surgical models.

- The introduction of ISBCS can potentially reduce costs to the public health without compromising patient safety.

\section{How might these results change the focus of} research or clinical practice?

The introduction of ISBCS into high volume surgical models as described in the paper require clinical evaluation.

is operated on at a time due to the perceived benefits of avoiding rare catastrophic bilateral sight-threatening complications such as endophthalmitis, ${ }^{34}$ as well as non-sight threatening complications such as the bilateral occurrence of refractive surprise. ${ }^{5}$ However, over the past few years, immediately sequential bilateral cataract surgery (ISBCS) has been being increasingly advocated. ${ }^{6}$ Studies have shown it to be safe with no cases of bilateral endophthalmitis reported in a series of 95606 cases and an overall postoperative endophthalmitis rate of 1 in 14352 cases with the routine use of intracameral antibiotics, ${ }^{7}$ while concerns of refractive surprise have been 
allayed by excluding patients with risk factors, ${ }^{8}$ identification and treatment of pre-existing ocular surface disease ${ }^{9}$ and the use of optical biometry and modern intraocular lens calculation formulae. ${ }^{10}$ It has been documented to be cost-effective compared with unilateral surgery in terms of hospital expenditure, ${ }^{11}$ and such cost efficiencies are greater when patient travel, paid home care costs and loss of working time are taken into consideration. ${ }^{12} 13$

With an increasingly aged population, population growth, increasing patient expectations and growing rates of age-related chronic diseases associated with cataracts, such as diabetes, the demand for cataract surgery over the past decades has continued to rise rapidly ${ }^{14}$ and is likely to further increase. Worldwide, a great deal, if not most, of this increasing demand for cataract surgery will have to be met within the public health sector. In 2014-2015, over 370000 cataract operations were performed by the National Health Service (NHS) in the UK. ${ }^{15}$ This was almost four times the number performed in 1989 , with cataract surgery being the most common surgical procedure being undertaken in the NHS ${ }^{16}$ With current economic and increasing resource constraints, the increased future demand for cataract surgery is likely to be problematic. Meeting such an ever-greater demand within the public health sector will require significant improvements in efficiency with the development of high-volume surgical models, while maintaining and trying to improve standards of patient care.

While ISBCS has been shown to be cost-effective in terms of inpatient care ${ }^{11}$ and patient travel, home care costs and loss of working time, ${ }^{1213}$ it also offers the ability to improve operating room (OR) efficiency, in terms of reduced patient OR transfer times and patient preparation times within the OR, while still assuming that all patient checks, documentations, preparations of surgical trollies and draping are carried out unilaterally, with separate instrumentation, and so on so that patient safety is not compromised. These improvements in OR time efficiency have the potential of improve OR efficiency by allowing more cataract cases to be surgically treated within a given time.

We have recently undertaken and published time and motion (TMS) studies of cataract surgery within the
OR in different public health Sector (NHS) settings, ${ }^{17}$ precisely documenting patient transfer, preparation and surgical times within the OR, as well as studying the costings of both conventional phacoemulsification cataract surgery and femtosecond laser-assisted cataract surgery in NHS settings. ${ }^{18}$ Using this data, in this current study we modelled the perceived time advantages of undertaking ISBCS and whether such time efficiencies could improve OR surgical efficiency by allowing more cases to be undertaken with a typical 4-hour NHS OR session.

\section{METHODS}

We have previously published continuous observation TMS in seven different routine 4-hour cataract OR sessions in five different public sector institutions (table 1). These cataract sessions included 'routine' lists in two district general hospitals and two teaching hospitals, a weekend waiting-list initiative session and a dedicated 'high-volume' theatre list in a teaching hospital and an NHS cataract surgery list in a private 'day-case' hospital. ${ }^{17}$ The five settings investigated were Guy's and St Thomas' NHS Foundation Trust, Southend University Hospital NHS Foundation Trust, the West Suffolk NHS Foundation Trust, the Norfolk and Norwich NHS Foundation Trust and the BMI Southend Private Hospital ${ }^{17}$ (table 1). Eighteen separate cataract OR sessions were observed, comprising 140 separate surgeries and all sessions were observed between 2 and 4 times. ${ }^{17}$ All surgeries were cataract only and conducted under local anaesthesia by phacoemulsification and all were unilateral surgeries. ${ }^{17}$ All surgeries were undertaken by a consultant or associate specialist ophthalmic surgeon, with none being designated teaching lists.

This was an observational study of medical personnel tasks and the timings of these tasks and patients were not directly involved in the study. The methodology of the collection of our TMS data has been published previously. ${ }^{17}$ Each list was observed by one or two ophthalmologists using a template Excel version 15 spreadsheet (Microsoft Corp, Redmond, Washington, USA) with specifically designed macros to facilitate the prompt and accurate recording of individual tasks undertaken within the OR and their timings. All tasks had been defined and

\begin{tabular}{|c|c|c|c|c|c|}
\hline Institution & 1 & 2 & 3 & 4 & 5 \\
\hline Type of OR list studied & Routine list & Routine list & $\begin{array}{l}\text { A. Routine list } \\
\text { B. Weekend initiative list } \\
\text { C. Dedicated high-volume list }\end{array}$ & Routine list & $\begin{array}{l}\text { High-volume list with NHS } \\
\text { patients receiving surgery } \\
\text { at private institution }\end{array}$ \\
\hline $\begin{array}{l}\text { No. of sessions } \\
\text { observed }\end{array}$ & 4 & 4 & $\begin{array}{l}\text { A. } 2 \\
\text { B. } 2 \\
\text { C. } 2\end{array}$ & 2 & 2 \\
\hline $\begin{array}{l}\text { Average no. of } \\
\text { operations scheduled/ } \\
\text { list }\end{array}$ & 6 & 6 & $\begin{array}{l}\text { A. } 7.5 \\
\text { B. } 9 \\
\text { C. } 13.5\end{array}$ & 7 & 13 \\
\hline
\end{tabular}

NHS, National Health Service; OR, operating room; TMS, time and motion. 


\section{Box 1 List of define tasks for TMS analysis}

Defined tasks for TMS analysis
Patient time in theatre
Time between cases
Time from patient entering theatre to start of operation
Time for patient to exit theatre after operation
Surgical time
Time surgeon spends on paperwork
Surgeon scrub time
Nurse scrub time
Nurse time to prepare scrub trolley
Nurse time to prepare phacoemulsification machine
Nurse time to clear equipment
Time spent on WHO checklist
TMS, time and motion.

agreed in terms of their relevance and start and finish times to maintain reproducibility of data collection (table 1) ${ }^{14}$ The tasks and their timings included: patient time in OR (defined as the time of admission to the OR until the time to exit from the OR); time between cases (defined as the time between one patient leaving the OR and the next patient entering the OR); patient entry time (time from patient admission into OR until end of positioning for surgery); patient exit time (time from removal of lid speculum to the patient exiting from the OR); surgical start and end times (defined as the point of insertion and removal of lid speculum); scrubbing time (time from opening of the tap and finishing gowning); nurse time to prepare scrub trolley (defined as time from opening first pack to time to beginning of preparation of up phacoemulsification machine); nurse time to prepare phacoemulsification machine; Safety/WHO checklist time (time once the first member of staff began speaking until the last member of staff had finished speaking); scrub nurse clearing up time (time when the first instrument was passed out or dismantled once lid speculum had been removed to when the scrub nurse re-entered the theatre from the sluice after disposing of all equipment and waste) and surgeon paperwork time (defined as the start of the surgeon writing the operation notes/ signing WHO check list to finish, Box 1). ${ }^{17}$

These timings were used to model the introduction of ISBCS cases into the OR sessions, with references to any possible time savings in terms of time between cases and patient entry time and patient exit times, while taking into account the fact that each cataract operation, although bilateral, would be undertaken, for safety considerations, as a totally separate procedure with rescrubbing of the surgeon and scrub nurse, the set-up of new scrub trollies and phacoemulsification equipment and repeat of Safety/WHO checklists. It is of note however, that, in the case of these latter tasks, some are performed simultaneously by different members of staff and hence timings will overlap. The modelling therefore assumed that while the first eye of ISBCS is being undertaken, a separate scrub nurse will scrub and prepare the trolley for the second eye, a factor which is also assumed in our model for unilateral surgeries. Therefore, the time required to switch between finishing the first eye and starting the second eye of any ISBCS is assumed to comprise of surgeon paperwork time, Safety/WHO checklist time and surgeon scrub time.

Modelling was undertaken both for each separate session in all settings, and for all settings as a whole. Possible efficiencies were calculated in terms of time taken to perform two cataract cases, percentage increase in numbers of eyes operated per 4-hour theatre session, the efficiency quotient (defined as the proportion of time that the surgeon was engaged in a task that is, total surgeon time spent productive/total time),${ }^{19}$ the surgery quotient (defined as the proportion of time that surgery was occurring, that is, total surgical time/total time) and the theatre utilisation quotient (defined as the utilisation of the maximum available theatre time, that is, time between start of first and end of last case/4hours).$^{20}$

\section{Statistics}

Data are presented as non-parametric and parametric as appropriate. Descriptive statistics were used to calculate averages and SD of the performances in each list. IBM SPSS Statistics for Windows (V.22.0, IBM Corp, Armonk, New York, USA) was used to perform analysis.

\section{RESULTS}

Based on the averaged timings for the defined tasks analysed by our TMS study in Box 1, for all seven separate cataract OR sessions across the five NHS settings studied, our modelling demonstrated that with the introduction of ISBCS it is possible to achieve an average $16 \%$ reduction in the time taken to perform two cataract surgeries (table 2, figure 1). Assuming that the average time taken to switch between finishing the first eye and starting the second eye of any ISBCS was comprised of Surgeon paperwork time, Safety/WHO checklist time and Surgeon scrub time and that during this time the Scrub nurse would be preparing the phacoemulsification machine, the average time in the OR to complete a single ISBCS case was $35.12 \mathrm{~min}$, compared with $41.74 \mathrm{~min}$ for two unilateral cases (Box 1, figure 1). Therefore, based on these figures, in a 4-hour OR session it is maximally possible to complete six separate ISBCS procedures (12 eyes) plus a single unilateral case, that is, 13 cases in total. This allows for a potential maximal efficiency quotient with ISBCS of $72 \%$, a surgery quotient of $57 \%$ and a theatre utilisation quotient of $95 \%$. Compared with the average number of 8.86 cases booked and undertaken per 4-hour OR session for the seven lists we studied (table 1), it is possible to achieve a $46.7 \%$ efficiency improvement with ISBCS. Indeed, even compared with the maximum number of cases possible per 4-hour list based on our averaged timings of 11 unilateral surgeries (table 2), an $18.2 \%$ efficiency improvement with ISBCS is still achievable. Based on the average figures, to be able to achieve enough time savings to conduct an extra unilateral case, 


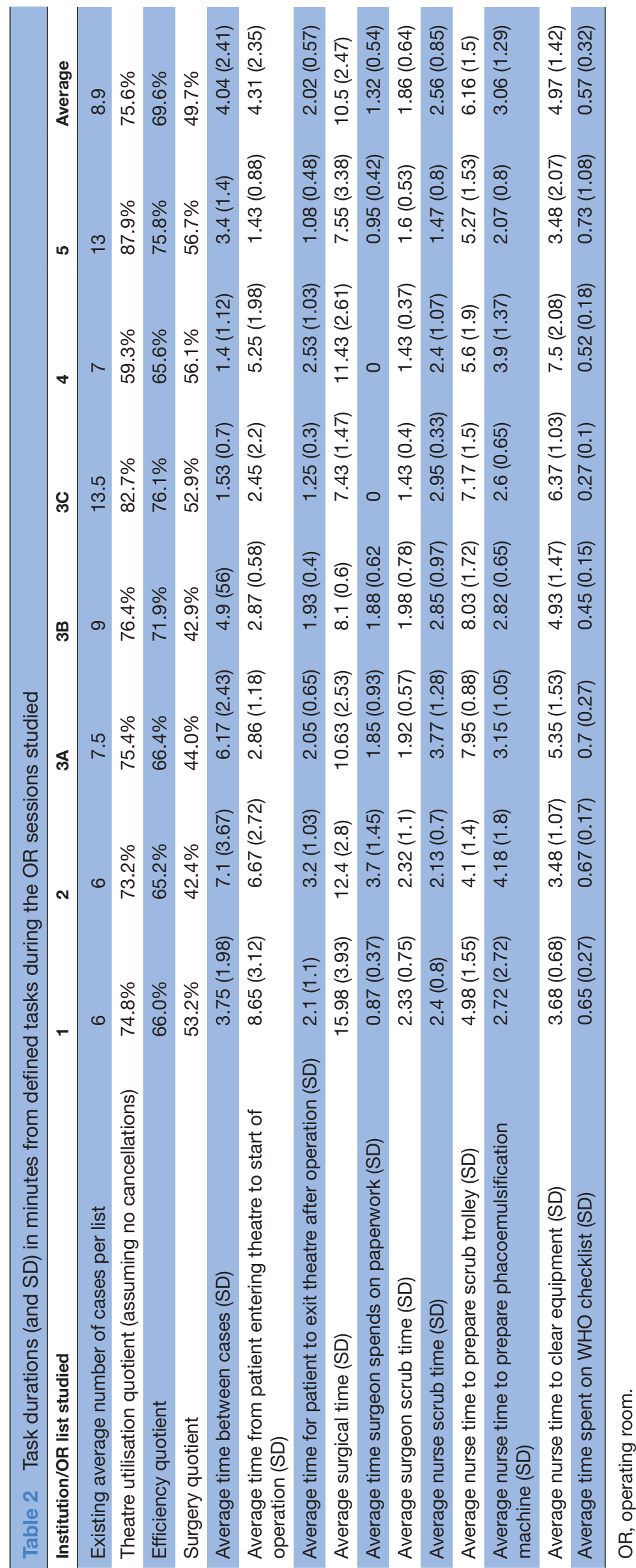




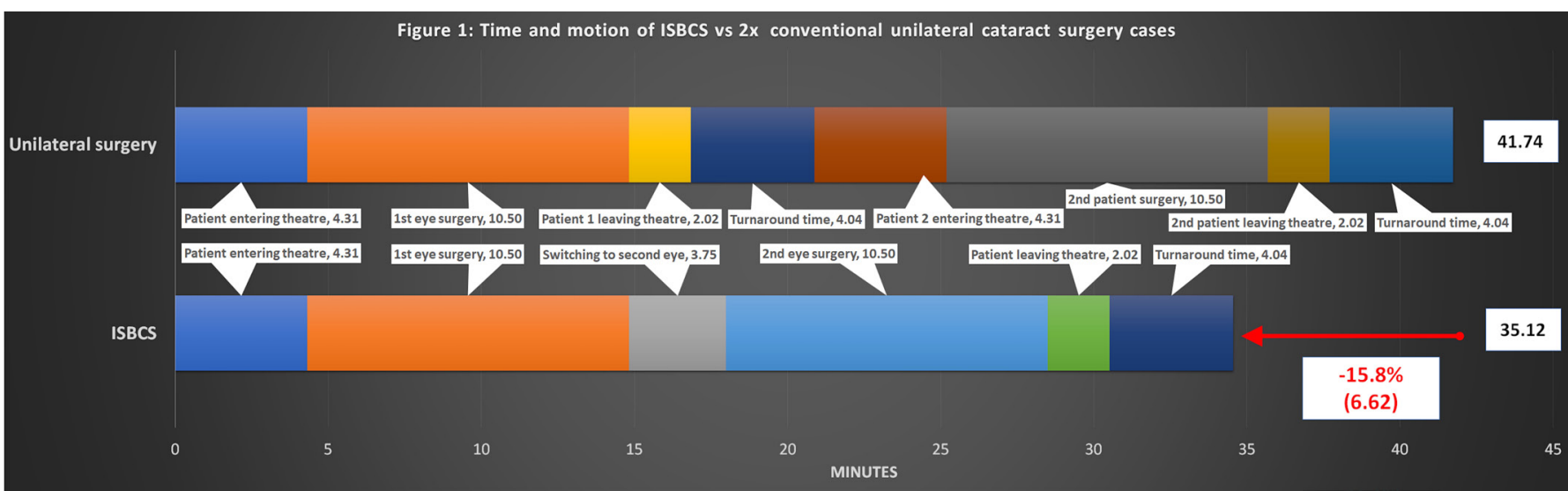

Figure 1 Time and motion of ISBCS versus two conventional unilateral cataract surgery cases based on averaged figures for all 7 OR session studies. ISBCS, immediately sequential bilateral cataract surgery; OR, operating room.

four ISBCS cases need to be undertaken on a 4-hour OR list.

In terms of individual OR sessions, for setting 1 (table 1), a routine cataract list within an NHS hospital, the time taken to complete a single ISBCS case was $50.1 \mathrm{~min}$, compared with $60.96 \mathrm{~min}$ for two unilateral cases (table 2), representing a $17.8 \%$ reduction in the time taken to undertake two cataract surgeries. In a 4-hour OR session in this setting it is maximally possible to complete four separate ISBCS procedures (eight eyes) plus a single unilateral case, that is, nine cases in total compared with the median of six cases booked for this list (table 1) and a maximal seven possible unilateral cases (table 3). This represents a possible $50 \%$ (cases actually booked) and $28 \%$ (maximum cases possible) efficiency improvements in cases undertaken respectively and allows for a maximum efficiency quotient with ISBCS of $74 \%$, a surgery quotient of $60 \%$ and a theatre utilisation quotient of $96 \%$. For setting 1 , based on the current key task timings, to be able to achieve enough time savings to conduct an extra unilateral case, four ISBCS cases need to be undertaken.
For setting 2 (table 1 ), a routine cataract list within an NHS hospital, the time taken to complete a single ISBCS case was $48.46 \mathrm{~min}$, compared with $58.74 \mathrm{~min}$ for two unilateral cases (table 2), representing a $17.5 \%$ reduction in the time taken to undertake two cataract surgeries. In a 4-hour OR session in this setting, it is maximally possible to complete four separate ISBCS procedures (eight eyes) plus a single unilateral case, that is, nine cases in total compared with the median of six cases booked for this list (table 1) and a possible maximum of eight unilateral cases (table 3). This represents possible $50 \%$ (cases actually booked) and $12.5 \%$ (maximum cases possible) efficiency improvements in cases undertaken and allows for a maximum efficiency quotient with ISBCS of $71.6 \%$, a surgery quotient of $46.5 \%$ and a theatre utilisation quotient of $93 \%$. For setting 2, based on the current key task timings, to be able to achieve enough time savings to conduct an extra unilateral case, three ISBCS cases need to be undertaken.

For setting $3 \mathrm{~A}$ (table 1 ), a routine cataract list within an NHS hospital, the time taken to complete a single ISBCS case was $36.81 \mathrm{~min}$, compared with $43.42 \mathrm{~min}$

Table 3 Existing and theoretical numbers of cases per list with ISBCS and unilateral surgery and possible percentage improvement in productivity

\begin{tabular}{|c|c|c|c|c|c|c|c|}
\hline Institution/OR list studied & 1 & 2 & 3A & 3B & $3 C$ & 4 & 5 \\
\hline Existing number of cases per list & 6 & 6 & 7.5 & 9 & 13.5 & 7 & 13 \\
\hline Maximum no. of unilateral cases possible & 7 & 8 & 11 & 13 & 18 & 11 & 17 \\
\hline $\begin{array}{l}\text { Maximum number of ISBCS cases possible } \\
\pm 1 \text { unilateral case }\end{array}$ & $4(+1)=9$ & $4(+1)=9$ & $6=12$ & $7(+1)=15$ & $11=22$ & $7=14$ & $9=18$ \\
\hline $\begin{array}{l}\text { Gain in no. of eyes with maximal no. with ISBCS } \\
\text { compared with existing cases }\end{array}$ & 3 & 3 & 5 & 6 & 8.5 & 7 & 5 \\
\hline $\begin{array}{l}\text { Improvement in productivity \% compared with } \\
\text { current no. per list }\end{array}$ & $50 \%$ & $50 \%$ & $60 \%$ & $67 \%$ & $63 \%$ & $50 \%$ & $38 \%$ \\
\hline $\begin{array}{l}\text { Improvement in productivity \% compared with } \\
\text { maximum no. of unilateral case per list }\end{array}$ & $28 \%$ & $12.5 \%$ & $9 \%$ & $15.4 \%$ & $22 \%$ & $27 \%$ & $12 \%$ \\
\hline $\begin{array}{l}\text { No. of ISBCS cases required to allow an extra } \\
\text { unilateral case to be performed }\end{array}$ & 4 & 3 & 4 & 4 & 3 & 3 & 6 \\
\hline
\end{tabular}

ISBCS, immediately sequential bilateral cataract surgery. 
for two unilateral cases (table 2), representing a $15.2 \%$ reduction in the time taken to undertake two cataract surgeries. In a 4-hour OR session in this setting it is maximally possible to complete six separate ISBCS procedures (12 eyes) compared with the median of 7.5 cases booked for this list (table 1) and a possible maximum of 11 unilateral cases (table 3). This represents possible $60 \%$ (cases actually booked) and 9\% (maximum cases possible) efficiency improvements in cases undertaken and allows for a maximum efficiency quotient with ISBCS of $75.5 \%$, a surgery quotient of $53 \%$ and a theatre utilisation quotient of $92 \%$. For setting $3 \mathrm{~A}$, based on the current key task timings, to be able to achieve enough time savings to conduct an extra unilateral case, 4 ISBCS cases need to be undertaken.

For setting 3B (table 1), a weekend waiting-list initiative cataract list within an NHS hospital, the time taken to complete a single ISBCS case was $30.21 \mathrm{~min}$, compared with $35.6 \mathrm{~min}$ for two unilateral cases (table 2), representing a $15.1 \%$ reduction in the time taken to undertake two cataract surgeries. In a 4-hour OR session in this setting it is maximally possible to complete seven separate ISBCS procedures (14 eyes) plus a single unilateral case, that is, 15 cases in total compared with the median of nine cases booked for this list (table 1) and a possible maximum of 13 unilateral cases. This represents possible $66.6 \%$ (cases actually booked) and $15.4 \%$ (maximum cases possible) efficiency improvements in cases undertaken and allows for a maximum efficiency quotient with ISBCS of $77.6 \%$, a surgery quotient of $50.6 \%$ and a theatre utilisation quotient of $95.5 \%$. For setting 3B, based on the current key task timings, to be able to achieve enough time savings to conduct an extra unilateral case, four ISBCS cases need to be undertaken.

For setting 3C (table 1), a dedicated high-volume list in an NHS hospital, the time taken to complete a single ISBCS case was $21.79 \mathrm{~min}$, compared with $26.24 \mathrm{~min}$ for two unilateral cases (table 2), representing a $16.9 \%$ reduction in the time taken to undertake two cataract surgeries. In a 4-hour OR session in this setting it is maximally possible to complete 11 separate ISBCS procedures (22 eyes) compared with the median of 13.5 cases booked for this list (table 1) and a possible maximum of 18 unilateral cases (table 3). This represents possible $63 \%$ (cases actually booked) and 22\% (maximum cases possible) efficiency improvements in cases undertaken and allows for a maximum efficiency quotient with ISBCS of $83.7 \%$, a surgery quotient of $68.1 \%$ and a theatre utilisation quotient of $99.8 \%$. For setting $3 \mathrm{C}$, based on the current key task timings, to be able to achieve enough time savings to conduct an extra unilateral case, three ISBCS cases need to be undertaken.

For setting 4 (table 1 ), a routine cataract list within an NHS hospital, the time taken to complete a single ISBCS case was $33.99 \mathrm{~min}$, compared with $41.22 \mathrm{~min}$ for two unilateral cases (table 2), representing a $17.5 \%$ reduction in the time taken to undertake two cataract surgeries. In a 4-hour OR session in this setting it is maximally possible to complete seven separate ISBCS procedures (14 eyes) compared with the median of seven cases booked for this list (table 1) and a possible maximum 11 unilateral cases (table 3). This represents possible 50\% (cases actually booked) and 27\% (maximum cases possible) efficiency improvements in cases undertaken and allows for a maximum efficiency quotient with ISBCS of $78 \%$, a surgery quotient of $66.7 \%$ and a theatre utilisation quotient of $99.1 \%$. For setting 4 , based on the current key task timings, to be able to achieve enough time savings to conduct an extra unilateral case, three ISBCS cases need to be undertaken.

For setting 5 (table 1), a high-volume list with NHS patients receiving surgery at private institution, the time taken to complete a single ISBCS case was $24.29 \mathrm{~min}$, compared with $26.92 \mathrm{~min}$ for two unilateral cases (table 2), representing a $9.8 \%$ reduction in the time taken to undertake two cataract surgeries. In a 4-hour OR session in this setting it is maximally possible to complete nine separate ISBCS procedures (18 eyes) compared with the median of 13 cases booked for this list (table 1) and a possible maximum 17 unilateral cases (table 3). This represents possible 38\% (cases actually booked) and $11.7 \%$ (maximum cases possible) efficiency improvements in cases undertaken and allows for a maximum efficiency quotient with ISBCS of $85.7 \%$, a surgery quotient of $59.8 \%$ and a theatre utilisation quotient of $96.7 \%$. For setting 5 , based on the current key task timings, to be able to achieve enough time savings to conduct an extra unilateral case, six ISBCS cases need to be undertaken.

\section{DISCUSSION}

TMS, introduced over a century ago, is a technique combining the time study work of Frederick Winslow Taylor with the motion study work of Frank and Lillian Gilbreth. It was developed as a scientific methodology to investigate the management of workers, in order to improve industrial productivity. Traditionally it was applied to manufacturing industry but has been shown to be of value within healthcare services. ${ }^{21}{ }^{22}$ In our previous TMS of cataract surgery within the NHS, we documented substantial inconsistencies in the number of cases performed per 4-hour list with medians of 6 to 13.5 cases, in the timings for key tasks to be undertaken and in efficiencies of patient flow between different institutions and OR settings. ${ }^{17}$ We identified requirements and factors for high-volume cataract surgery models, specifically the support of cataract surgeons with sufficient allied healthcare professionals (AHPs), within and outside the OR, and with specific tasks normally performed by the surgeons, such as completing paperwork, assisting with patient transition and instilling anaesthetic drops, and so on, being undertaken by AHPs that could improve surgical efficiency in terms of numbers of cases performed in a 4 -hour period by over $100 \%$ in some settings. ${ }^{17}$

In this study, all 140 cases documented were unilateral. ${ }^{17}$ We hypothesised that further efficiencies might be 
achieved over those already identified with the introduction of ISBCS. In addition to being cost-effective in terms of hospital care $^{11}$ and costs relating to patient travel, home care and loss of working time, ${ }^{12}{ }^{13}$ we postulated that by reducing patient OR transfer times and patient surgical preparation times within the OR, time saving efficiencies were possible and if sufficient, they might allow extra cases to be performed in a given OR session or allow current surgical cases to be undertaken with less OR time.

Our modelling supports our hypothesis. The time taken to perform ISBCS was on average 16\% (range $9.8 \%-17.8 \%$ ) less than that required to undertake two unilateral cases, representing an average time saving of $6.62 \mathrm{~min}$ (range $2.63-10.59 \mathrm{~min}$ ). This translated into a mean 54\% improvement (range 38\%-67\%) in number of cases currently performed per list and an $18 \%$ improvement (range 9\%-28\%) even if the number of unilateral cases per list had been fully maximised (table 3). It also indicated that an average number of four ISBCS cases per list (range 3-6) were required to achieve sufficient time savings to allow an extra unilateral surgery to be conducted (table 3 ).

There are a number of caveats to our study. Because TMS by its nature is very time consuming, the scope of our original TMS study was somewhat limited, 140 separate operations in 18 OR sessions. However, it was the first of its kind in cataract surgery ${ }^{17}$ and we deliberately conducted it in different NHS settings, including a mixture of university and district general hospitals, with rural and urban populations and varying cataract surgical lists, both routine and so-called 'high-volume', to try and provide an overview of NHS cataract service provision models.

Interestingly with ISBCS, our modelling indicated that efficiencies were possible in all the studied OR settings, even including the more efficient 'high-volume' OR lists such as setting 3C (tables 1-3), where 13.5 cases per 4-hour list were routinely booked, but a maximal 11 ISBCS cases (22 eyes) theoretically possible, representing a $63 \%$ improvement in productivity (table 3 ). In this context, our remodelling for ISBCS appears to support the widespread introduction of ISBCS in the state-funded healthcare system in the UK to help improve surgical efficiency. However, it does perhaps raise one interesting issue with reference to surgeon fatigue in very highvolume lists, such as setting 4 where theoretically 22 cases can be completed in a 4-hour period. Although this has not been demonstrated in the past, ${ }^{23}$ it probably requires further investigation and perhaps the development of new 'high-volume' surgery delivery models such as split surgeon OR sessions, to negate possible fatigue problems, similar to current practices for pilots in the commercial airline industry.

We found that depending on the unit, an average of four ISBCS cases (range 3-6) were required to allow sufficient time savings for one extra unilateral case to be undertaken (table 3). Clearly undertaking less than the required number of ISBCS per list will not introduce any efficiencies with regards to the OR productivity, although there will still be other cost benefits outside the OR to both the patient and hospital, that need to be considered. ${ }^{11-13}$ Therefore, for such OR efficiency models to work, the requisite number of ISBCS cases need to be booked into a specific OR session with the correct mix of ISBCS and unilateral cases to maximise efficiency (or in the case of very efficient units, such as setting $3 \mathrm{C}$, where time efficiencies can allow extra ISBCS cases to be undertaken, further patients who have to undergo ISBCS). This is likely to mean that only specific, select cataract surgery lists in any particular setting will contain ISBCS cases in order to maximise OR productivity. This is also important as many patients may not be suitable for or wish to undergo ISBCS and there should be no waiting-list delay for unilateral cases at the expense of the introduction of ISBCS into the public sector. It also means that there has to be sufficient numbers of ISBCS cases to support such specialised ISBCS lists, with sufficient doctors willing to undertake ISBCS and sufficient patients who are suitable and then willing to undergo ISBCS. In order to address this within the NHS, we are currently conducting a further study addressed at patient ISBCS preferences to investigate whether sufficient numbers of patients wish to undergo and are suitable for ISBCS to support such OR models. Indeed, patient selection is an important factor for ISBCS, being best performed in 'routine' cases, with no pre-existing ocular disease ${ }^{89}$ or previous ocular surgery, ${ }^{8}$ who are at low risk of ocular complications during and after surgery, who have sufficient home support and accurate and reproducible optical biometric measurements. ${ }^{10}$

Other limitations are basically related to those of the original TMS study. ${ }^{17}$ We only evaluated local anaesthetic models of surgical provision and did not include OR sessions with sedation or general anaesthetic (GA) cases. This was because, the vast majority of cataract surgery performed in the UK is conducted under topical/local anaesthesia, ${ }^{1}$ and the aim of the TMS study was to focus on the delivery of high-volume cataract services, where GA cases are unlikely to feature. ${ }^{17}$ TMS studies specifically investigating cataract surgery lists with GA provision are required to determine if the introduction of ISBCS cases offer time efficiencies in such circumstances to allow extra cases to be undertaken, although given the additional anaesthetic administration and recovery times associated with GA and sedation it is very likely that time efficiencies with ISBCS are possible and likely to be greater per case compared with local anaesthetic surgery, notwithstanding the risks of death or serious complication from GA, estimated at 7.3/1 000000 (for each GA in an American Society of Anesthesiologists (ASA) grade 1 or 2) ${ }^{24}$ which may outweigh the risk of bilateral simultaneous endophthalmitis. ${ }^{6}$

The original TMS study also focused on consultant or associate specialist surgeon theatre sessions rather than on 'training' lists with junior doctors. ${ }^{17}$ Obviously, there 
is requirement to provide both high-quality training for the next generation of surgeons and high-volume surgical provision models to address the increasing demands for cataract surgery. ${ }^{14-16}$ However, as previously discussed, ${ }^{13}$ this is possible with separately designated 'high' volume and 'teaching' OR sessions. In addition, given the potential cost benefits it seems entirely sensible to introduce junior doctors to the concept of ISBCS, as well as high-volume surgery, in their training, while of course maintaining appropriate standards to maintain patient safety.

One of the factors limiting the introduction of ISBCS into the NHS is the current reimbursement system, whereby payment for second eye surgery when it is conducted simultaneously is significantly discounted and often not reimbursed at all, making it financially punitive. Given the published evidence of the cost-efficiency of ISBCS in terms of hospital/clinic care, with reduced appointments (preoperative assessment and follow-up visits) and patient waiting time for second eye surgery ${ }^{11}$ as well as cost-efficiencies to patients themselves such as travel time and costs, home care costs and loss of working time, ${ }^{12} 13$ added to the efficiencies within the OR highlighted by this current study, this appears to be imprudent. If ISBCS is cost-effective and further OR efficiencies can be achieved, then it is sensible that non-punitive tariffs be considered, so that tax-payers' money is spent effectively within the public healthcare sector. Indeed, as discussed above, with TMS data it is possible to determine how many ISBCS cases are required on a particular list, in a particular NHS setting to allow an extra unilateral surgery case to be conducted and thereby offer reduced tariffs for the second eye in ISBCS that are not punitive.

\section{CONCLUSIONS}

This current study, by remodelling data from a previous TMS of cataract surgery, indicates that the introduction of routine ISBCS has the potential to improve the productivity of cataract surgery within the NHS, with efficiencies being possible in both high-volume and low-volume surgical models. It highlights the power of TMS studies to identify factors for improving surgical efficiency and in this particular study the number of ISBCS cases per individual OR session required to achieve surgical efficiencies and hence the ability for such TMS models to set tariffs for surgical reimbursement for ISBCS at reduced costs to the public health sector.

Acknowledgements The authors would like to thank BMI Southend Private Hospital, Norfolk and Norwich NHS Foundation Trust, Guy's and St Thomas' NHS Foundation Trust, Southend University Hospital NHS Foundation Trust and West Suffolk NHS Foundation Trust. The authors also thank Karen Bateman BSc IMM, Director, Tranoca Ltd; Ted Burton, Consultant Ophthalmic Surgeon, Norfolk and Norwich NHS Foundation Trust; Hosam Kasaby, Consultant Ophthalmic Surgeon, Southend University Hospital NHS Foundation Trust; James Myerscough, Consultant Ophthalmic Surgeon and Southend University Hospital NHS Foundation Trust.

Contributors DPO'B developed the concept of the paper. DPO'B and HR undertook data analysis. DPO'B, HR, KN and JG contributed to the writing of the manuscript.
Funding The authors have not declared a specific grant for this research from any funding agency in the public, commercial or not-for-profit sectors.

Competing interests DO'B holds non-commercial research grants with Alcon Inc and Rayner Ltd. He has undertaken consultancy work for Alcon Inc, Avedro Inc, Sooft Italia S.P.A. and Sparca Inc in the past 36 months. HR has undertaken consultancy work for Alcon Inc in the past 18 months.

Patient consent for publication Not required.

Provenance and peer review Not commissioned; externally peer reviewed.

Data availability statement Data are available upon request.

Open access This is an open access article distributed in accordance with the Creative Commons Attribution Non Commercial (CC BY-NC 4.0) license, which permits others to distribute, remix, adapt, build upon this work non-commercially, and license their derivative works on different terms, provided the original work is properly cited, appropriate credit is given, any changes made indicated, and the use is non-commercial. See: http://creativecommons.org/licenses/by-nc/4.0/.

ORCID iD

David P 0'Brart http://orcid.org/0000-0002-1065-3166

\section{REFERENCES}

1 Day AC, Donachie PHJ, Sparrow JM, et al. The Royal College of ophthalmologists' national ophthalmology database study of cataract surgery: report 1, visual outcomes and complications. Eye 2015;29:552-60.

2 Laidlaw DA, Harrad RA, Hopper CD, et al. Randomised trial of effectiveness of second eye cataract surgery. Lancet 1998;352:925-9.

3 Kashkouli MB, Salimi S, Aghaee H, et al. Bilateral Pseudomonas aeruginosa endophthalmitis following bilateral simultaneous cataract surgery. Indian J Ophthalmol 2007;55:374-5.

4 Ozdek SC, Onaran Z, Gürelik G, et al. Bilateral endophthalmitis after simultaneous bilateral cataract surgery. J Cataract Refract Surg 2005;31:1261-2.

5 Olsen T. Use of fellow eye data in the calculation of intraocular lens power for the second eye. Ophthalmology 2011;118:1710-5.

6 Arshinoff SA, Strube YNJ, Yagev R. Simultaneous bilateral cataract surgery. J Cataract Refract Surg 2003;29:1281-91.

7 Arshinoff SA, Bastianelli PA. Incidence of postoperative endophthalmitis after immediate sequential bilateral cataract surgery. $J$ Cataract Refract Surg 2011;37:2105-14.

8 Lundström M, Dickman M, Henry Y, et al. Risk factors for refractive error after cataract surgery: Analysis of 282811 cataract extractions reported to the European Registry of Quality Outcomes for cataract and refractive surgery. J Cataract Refract Surg 2018;44:447-52.

9 Ho VWM, Stanojcic N, O'Brart NAL, et al. Refractive surprise after routine cataract surgery with multifocal IOLs attributable to corneal epithelial basement membrane dystrophy. J Cataract Refract Surg 2019;45:685-9.

10 Roberts TV, Hodge C, Sutton G, et al. Contributors to the vision eye Institute IOL outcomes registry. Comparison of Hill-radial basis function, Barrett universal and current third generation formulas for the calculation of intraocular lens power during cataract surgery. Clin Exp Ophthalmol 2018;46:240-6.

11 O'Brien JJ, Gonder J, Botz C, et al. Immediately sequential bilateral cataract surgery versus delayed sequential bilateral cataract surgery: potential hospital cost savings. Can J Ophthalmol 2010;45:596-601.

12 Leivo T, Sarikkola A-U, Uusitalo RJ, et al. Simultaneous bilateral cataract surgery: economic analysis; Helsinki simultaneous bilateral cataract surgery study report 2. J Cataract Refract Surg 2011;37:1003-8.

13 Rush SW, Gerald AE, Smith JC, et al. Prospective analysis of outcomes and economic factors of same-day bilateral cataract surgery in the United States. J Cataract Refract Surg 2015;41:732-9.

14 Minassian DC, Reidy A. Future sight loss UK (2): an epidemiological and economic model for sight loss in the decade 2010-2020, 2009. Available: http://www. rnib. org. uk/ sites/ default/ files/ FSUK 2. pdf

15 Department of Health. Hes online health episode statistics, 2015.

16 Black N, Browne J, van der Meulen J, et al. Is there overutilisation of cataract surgery in England? Br J Ophthalmol 2009;93:13-17.

17 Roberts HW, Myerscough J, Borsci S, et al. Time and motion studies of national health service cataract theatre Lists to determine strategies to improve efficiency. Br J Ophthalmol 2018;102:1259-67.

18 Roberts HW, Wagh VK, Mullens IJM, et al. Evaluation of a hub-andspoke model for the delivery of femtosecond laser-assisted cataract 
surgery within the context of a large randomised controlled trial. $\mathrm{Br} J$ Ophthalmol 2018;102:1556-63.

19 Harewood GC, Chrysostomou K, Himy N, et al. A "time-and-motion" study of endoscopic practice: strategies to enhance efficiency. Gastrointest Endosc 2008;68:1043-50.

20 Weinbroum AA, Ekstein P, Ezri T. Efficiency of the operating room suite. Am J Surg 2003;185:244-50.

21 Burke TA, McKee JR, Wilson HC, et al. A comparison of time-andmotion and self-reporting methods of work measurement. J Nurs Adm 2000;30:118-25.
22 Finkler SA, Knickman JR, Hendrickson G, et al. A comparison of work-sampling and time-and-motion techniques for studies in health services research. Health Serv Res 1993;28:577-97.

23 Salman W, Park J, Kersey TL, et al. Assessment of fatigue in intraocular surgery: analysis using a virtual reality simulator. Graefe's Arch Clin Exp Ophthal 72011;249:77-81.

24 Schiff JH, Welker A, Fohr B, et al. Major incidents and complications in otherwise healthy patients undergoing elective procedures: results based on 1.37 million anaesthetic procedures. $\mathrm{Br} J$ Anaesth 2014:113:109-21. 